\title{
An automated design flow for vibration-based energy harvester systems
}

\author{
Leran Wang, Tom J. Kazmierski, Bashir M. Al-Hashimi, \\ Steve P. Beeby and Dibin Zhu \\ School of Electronics and Computer Science \\ University of Southampton, UK \\ \{lw04r,tjk,bmah,spb,db05r\}@ecs.soton.ac.uk
}

\begin{abstract}
This paper proposes, for the first time, an automated energy harvester design flow which is based on a single HDL software platform that can be used to model, simulate, configure and optimise energy harvester systems. A demonstrator prototype incorporating an electromagnetic mechanical-vibrationbased micro-generator and a limited number of library models has been developed and a design case study has been carried out. Experimental measurements have validated the simulation results which show that the outcome from the design flow can improve the energy harvesting efficiency by $75 \%$.
\end{abstract}

\section{INTRODUCTION}

Over the last decade, a key design constraint of the embedded electronics in mobile applications has been the power consumption, mainly due to the limitations of battery life. As a result, system level low-power design techniques have attracted a large body of research [1]. Pervasive computing, un-intrusive integration of computation into our daily lives through sensing systems to enhance our activities, is widely believed to represent the next major market for next generation wireless electronics industry. One of the key challenges in pervasive computing research is energy harvesting, where the focus is to develop energy harvesters to power electronic devices, particularly in wearable and embedded sensors [2] where batteries are cumbersome or impractical. At present there are considerable and continuing research efforts worldwide to support the energy harvesting paradigm and selfpowered electronics. An energy harvester has normally three main components: the micro-generator which converts ambient environment energy into electrical energy, the power processing circuit which regulates the generated voltage, and the storage element (Fig. 1).

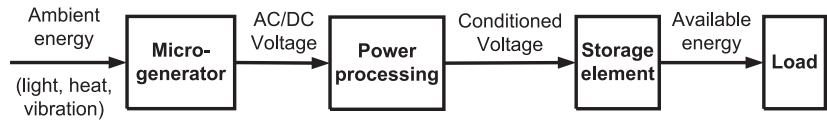

Fig. 1. Diagram of an energy harvester

The majority of the reported research in energy harvesting has been on improving the efficiency of the energy harvesters through the design and fabrication of novel micro-generators, materials and devices [3]. The amount of power that can be harvested in a particular application is highly dependent upon the energy source being harvested. Typically, power densities of around $800 \mu \mathrm{W} / \mathrm{cm}^{3}$ for machine vibration applications and up to $140 \mu \mathrm{W} / \mathrm{cm}^{3}$ for human powered applications can be expected [4], however, the power output of vibrationharvesting inertial generators is highly sensitive to the frequency and amplitude of the vibration source [5] and so these figures are indicative only. Practical generators have been reported with power densities of $17 \mu \mathrm{W} / \mathrm{cm}^{3}$ for a non-resonant device [6], [7], to a resonant device capable of generating $30 \mu \mathrm{W} / \mathrm{cm}^{3}$. Finite element analysis packages are being used to simulate and optimise the performance of the micro-generator part of the self-powered system [8]. Typically the generated voltage from a micro-generator is not suitable for powering an electronic device directly. For example the output voltage from an electromagnetic microgenerator is $\mathrm{AC}$ and too low while the piezoelectric device normally generates $\mathrm{AC}$ voltage that is too high. Therefore external analogue circuits are often employed to regulate (i.e boost up or step down, AC/DC conversion etc.) the voltage and store the energy in a battery or a super-capacitor. The importance of the energy storage element cannot be ignored. Super-capacitors, used to store the harvested energy, provide a significant challenge in terms of model development due to their complex performance loss characteristics caused by ageing and inappropriate discharging. To design and optimise the harvester associated electronics, simulators such as SPICE are often used. The micro-generator is usually modelled either as an ideal voltage source or an equivalent circuit model [9]. We have recently demonstrated through simulation and experimentation that such modelling technique of vibrationbased electromagnetic harvesters is inadequate for accurate prediction of the energy harvester performance [10]. A PSpice based electromechanical simulation tool kit for inertial microgenerators, which incorporates some additional features of the physical systems such as end stop dynamics and parasitic damping, has also been reported [11].

An energy harvester is a system consisting of several components from different physical domains including mechanical, magnetic and electrical as well as the external circuits which regulate and store the generated energy. To design highly efficient energy harvesters, we believe that it is crucial to consider the various parts of an energy harvester in the context 
of a complete system. As will be shown in this paper, the gain at one part may come at the price of efficiency loss elsewhere, rending the energy harvester much less efficient than expected. To date there has been no reported automated design flow for energy harvesters and the aim of this paper is to propose such an approach. A mixed-physical-domain hardware description language (HDL) can be used to model and correctly predict the performance of an energy harvester system. This is because the HDL describes the micro-generator and external electronics as an integrated model, so that the close mechanical-electrical interaction, which is often missing in traditional energy harvester design methods, can be captured accurately. Several HDLs that support multiple domain system modelling and simulation are available, such as VHDL-AMS, Verilog-AMS and SystemC-A. In this paper VHDL-AMS [12] has been chosen as the modelling language to demonstrate the concept of an automated energy harvester design flow.

\section{Proposed Energy harVester DESIGN Flow}

The input to the energy harvester design flow are the user specified micro-generator type (piezoelectric, electromagnetic, electrostatic) and the frequency/amplitude of excitation. Given a design objective, such as to achieve the best possible power density for specified micro-generator type to work at resonance, the output from the design flow are the dimension of micro-generator and suitable power processing circuits. The proposed design flow is outlined in the pseudo-code of Algorithm 1. Naturally, the process starts with initial design specification, such as available energy source, environmental energy density, device size, minimum voltage level/power output etc. According to these specifications, HDL models of energy harvester system components are constructed from component cells available in the component library. The component library contains parameterised models of different kind of micro-generator structures, various power processing circuit topologies and storage elements. The outer loop in the Algorithm 1 represents this structure configuration process, which involves examining and comparing those HDL models from the library with the aim of identifying a set of components that meet specific user requirements. The inner design flow loop will then find the best performance of each candidate design by adjusting electrical and non-electrical parameters of the design's mixed-technology HDL model. The parametric optimisation of the generated structure will further improve the energy harvester efficiency by employing suitable optimisation algorithms. Fig. 2 shows an implementation of the design flow in a VHDL-AMS testbench where the optimisation loop runs as a series of concurrent processes. The experimental results shown in Sections IV and V confirm the importance of both loops where the the design flow optimises mechanical and electrical parameters for each possible architecture and selects the one that extracts the most power from a given excitation. One of the key requirements for energy harvester component models is that models need to capture both theoretical equations and practical non-idealities required for accurate performance estimation. The models should support different mechanical-electrical structures and will be expressed in terms of HDL descriptions. They will be able to predict the behaviour of the actual device accurately while remaining reconfigurable. As proof of concept, a small VHDL-AMS model library has been built to demonstrate the efficiency of the design flow, as discussed in Section III. Based on the developed model library, Section IV describes the automated structure configuration that has been carried out using a single VHDL-AMS simulator. The configuration result and simulations of different energy harvester models have led to indepth understanding of how electromagnetic micro-generator performs when connected to lossy voltage multipliers to boost the micro-generator output. The results have been used for the performance optimisation, which is presented in Section V.
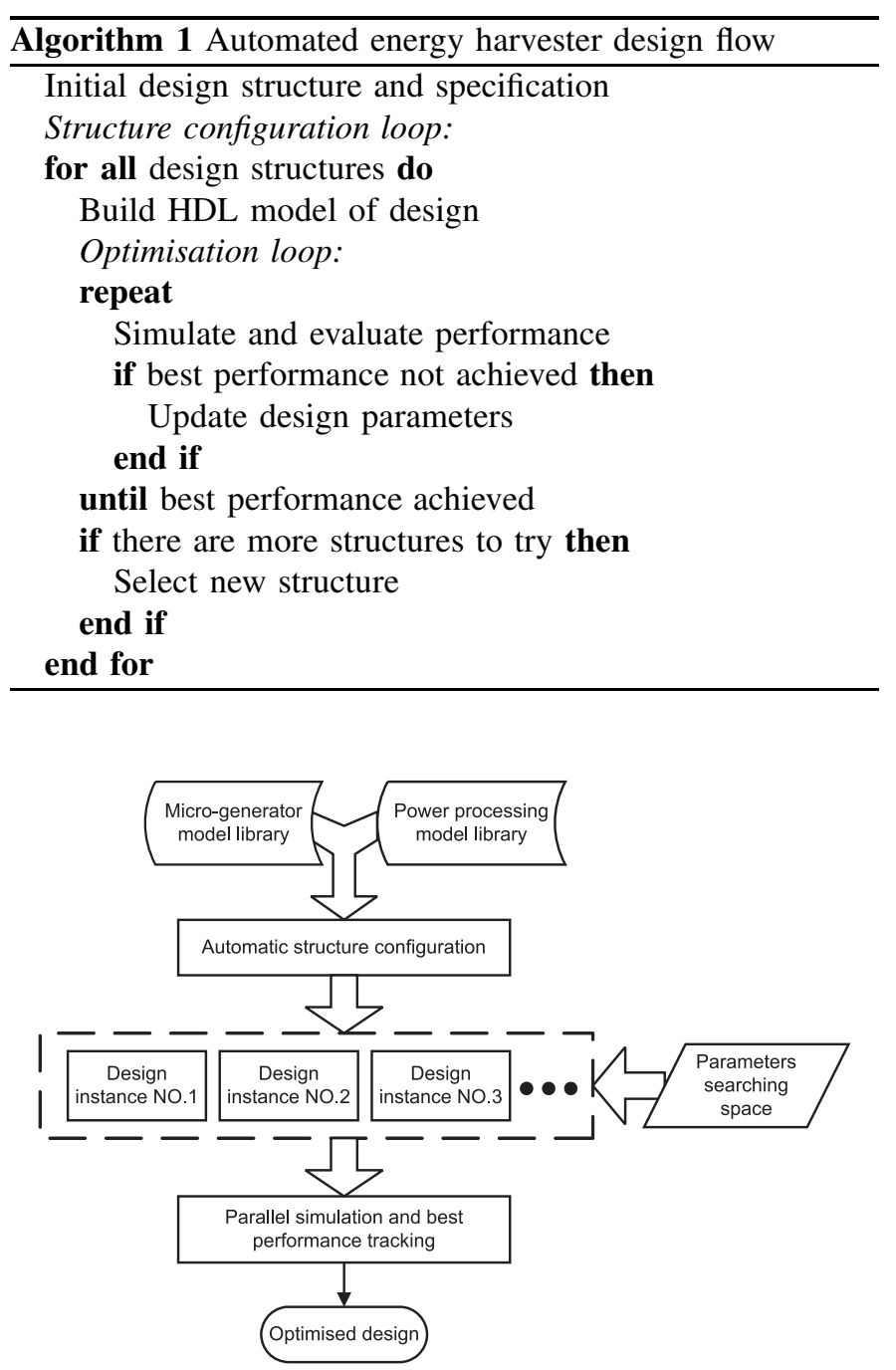

Fig. 2. Implementation of the proposed energy harvester design flow in VHDL-AMS

\section{ENERGY HARVESTER MODELS}

Vibration-based electromagnetic energy harvesters are proving to be successful when applied in real-world applications [13] and therefore are used in this paper as proof-of-concept 
for the proposed design flow. The case study presented in this paper uses a vibration-based electromagnetic micro generator [14] as an example. The design is based on a cantilever structure. The coil is fixed to the base and four magnets, which are located on both sides of the coil, form the proof mass (Fig. $3)$.

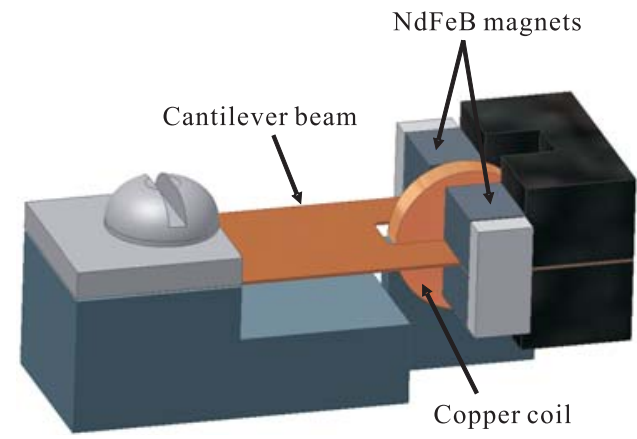

Fig. 3. Cantilever based electromagnetic micro-generator

This device can be modelled as a second-order springdamping system, which has been widely used [3], and whose dynamics is:

$$
m z \ddot{(t)}+c_{p} z \dot{(t)}+k_{s} z(t)+F_{e m}=-m y \ddot{(t)}
$$

where $m$ is the proof mass, $z(t)$ is the relative displacement between the mass and the base, $c_{p}$ is the parasitic damping factor, $k_{s}$ is the spring stiffness, $y(t)$ is the displacement of the base and $F_{e m}$ is the electromagnetic force. by:

The electromagnetic voltage generated in the coil is given

$$
\left.v_{e m}=\Phi * z \dot{z} t\right)
$$

where $\Phi=N B l$ is the magnetic flux through the coil and $N$ is the number of coil turns, $B$ is the magnetic field and $l$ is the effective length.

The output voltage is defined by:

$$
v(t)=v_{e m}-R_{c} * i(t)-L_{c} * i(\dot{t})
$$

where $R_{c}$ and $L_{c}$ are the resistance and inductance of the coil respectively and $i(t)$ is the current through the coil.

Finally, the electromagnetic force is calculated as:

$$
F_{\text {em }}=\Phi * i(t)
$$

In the above equations, the coil parameters are given in number of turns $N$ and resistance $R_{c}$. However, when manufacturing a coil, the specification is often given by the thickness $t$, inner radius $r$, outer radius $R$ and wire diameter $d$. To build parameterised HDL models, the relations between these parameters are incorporated and listed below.

The total wire length is:

$$
l=4 * f * t *\left(R^{2}-r^{2}\right) / d^{2}
$$

where $f$ is the fill factor.

The number of turns is:

$$
N=l /\left(2 * \pi * R_{\text {ave }}\right)
$$

where $R_{\text {ave }}=(R-r) / 2+r$ is the average radius.

The coil resistance is given by:

$$
R_{c}=4 * \rho * l /\left(\pi * d^{2}\right)
$$

where $\rho$ is the resistivity of the material.

Part of the VHDL-AMS model code is given below. The omitted part describes the actual magnetic flux through the coil $(\Phi)$ as a piecewise function of the relative displacement between the coil and magnet, details of which have been discussed in a previous paper [10].

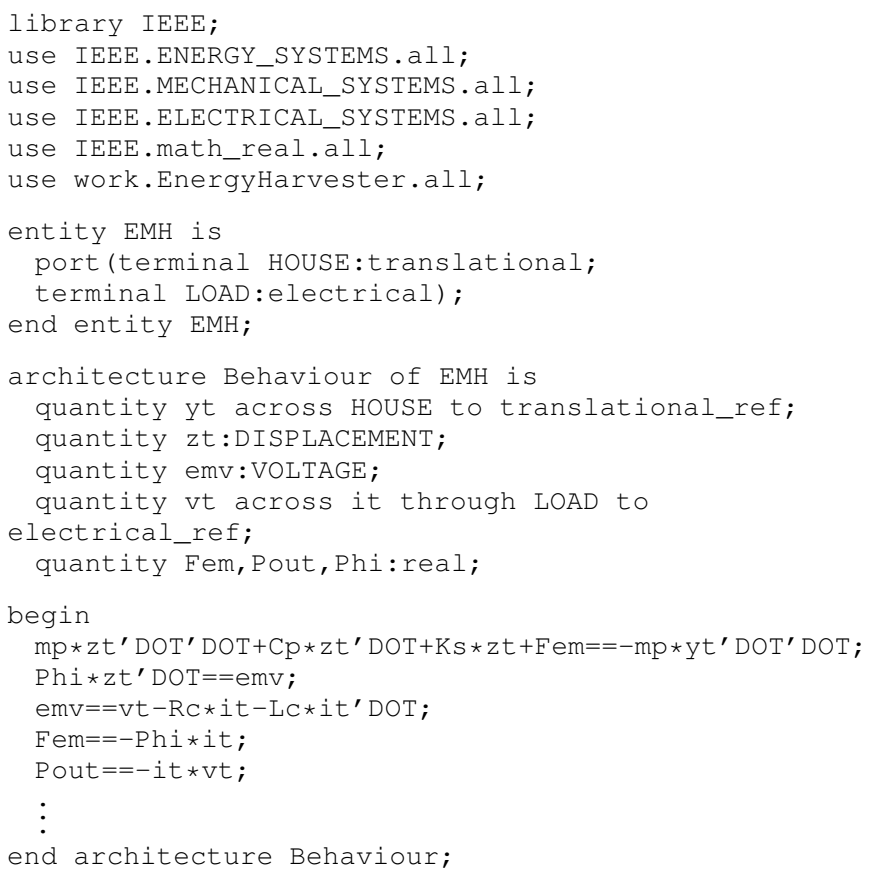

The above model correctly captures the practical nonidealities of the micro-generator by linking its non-linear mechanical and magnetic characteristics with the electrical output. Two types of this micro-generator have been manufactured and modelled, which are based on the same structure but have different dimensions. Some of the key parameters are listed in Table I. As can be seen from the table, microgenerator Type II is bigger than Type I and because the coil is changeable, both the micro-generators can have different wire diameters.

TABLE I

MICRO-GENERATORS PARAMETERS

\begin{tabular}{c|cc}
\hline & Type I & Type II \\
\hline Proof mass $(\mathrm{g})$ & 0.6 & 2.4 \\
\hline Magnet size $(\mathrm{mm})$ & 2.0 & 3.0 \\
\hline Cantilever length $(\mathrm{mm})$ & 10.0 & 13.7 \\
\hline Wire diameter(um) & $12 / 16 / 25$ & $16 / 25$ \\
\hline Coil radius $(\mathrm{mm})$ & 1.2 & 2.45 \\
\hline Coil thickness $(\mathrm{mm})$ & 0.48 & 1.3 \\
\hline
\end{tabular}


Standard voltage multipliers (VMs), which are used to rectify and boost up $\mathrm{AC}$ voltage, can act as the power processing circuit to an electromagnetic micro-generator in energy harvester systems. Fig. 4 shows two types of VM configuration, namely Villard (Fig. 4 (a) to (c)) and Dickson (Fig. 4 (d) to (f)) [15]. In our model library, the VMs are modelled on circuit-level in VHDL-AMS using standard RC components and diodes. 3, 4, and 5 stage VMs have been included in the library as shown in Fig. 4. Other types of booster configuration are also possible.

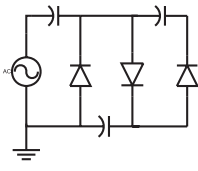

(a)

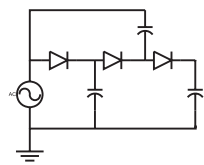

(d)

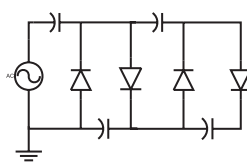

(b)

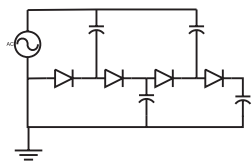

(e)

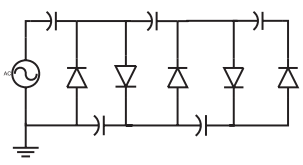

(c)

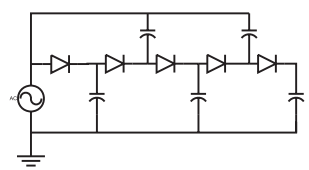

(f)
Fig. 4. Voltage multiplier configurations, (a)-(c): 3 to 5 stage Villard VM, (d)-(f): 3 to 5 stage Dickson VM

In case of the storage element, a super capacitor has been modelled as in Fig. 5 [16], where $R_{\text {leakage }}$ represents the leakage resistance and $R_{E S R}$ is the equivalent series resistance.

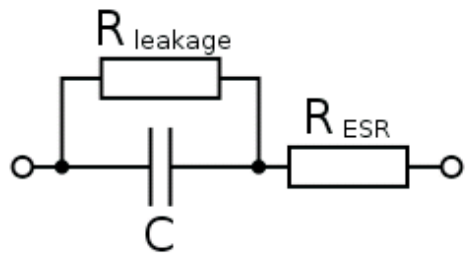

Fig. 5. Simplified super capacitor model

\section{AUtomatic STRUCTURE CONFIGURATION}

The automatic structure configuration process combines the outer loop in Algorithm 1, which selects an architectural structure of the whole energy harvester system, with parametric performance optimisation as discussed in the next section. The last section explains a small HDL model library of energy harvester components which contains two types of vibration micro-generator, each of which can be configured with different coils (wire diameter of 12/16/25 $\mu \mathrm{m}$ ), and two types of voltage boosters that have three to five stages. The configuration target has been set to find the set of components that can charge the $0.047 \mathrm{~F}$ super capacitor to $2 \mathrm{~V}$ in shortest time. Simulations of every possible energy harvester structure have been carried out simultaneously and a VHDL-AMS process has been developed and implemented in the testbench to automatically track the best model. The concurrent simulation of multiple configurations was enabled by the generate syntax in VHDL-AMS:

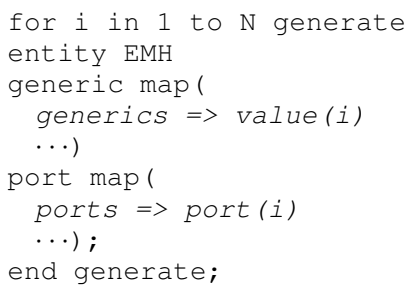

The SystemVision VHDL-AMS simulator from Mentor Graphics [17] has been used as the single software platform. Details of the outcome design are listed in Table II. The whole design flow based on Algorithm 1 took 26 hours of CPU time on a Pentium 4 machine.

TABLE II

PARAMETERS OF THE CONFIGURATION RESULT

\begin{tabular}{cc}
\hline Micro-generator & Type II \\
\hline Wire diameter & 25 um \\
\hline Voltage booster & 3-stage Dickson VM (Fig. 4(d)) \\
\hline
\end{tabular}

Not surprisingly, the micro-generator II has been chosen because it is bigger and stores more kinetic energy. However, it is interesting that the coil with the largest wire diameter, which leads to the fewest number of turns, and the VM with the fewest stages have been chosen. The integrated modelling approach in our design flow allows one to perform "what-if" scenarios. Therefore to further investigate the result, more simulations have been done and an important tradeoff between the electromagnetic micro-generator and the VM voltage booster has been found. Fig. 6 shows the charging waveforms of Type I micro-generator connected to the same 5stage VM but configured with different coils. At the beginning, the energy harvester with $25 \mu \mathrm{m}$ wire diameter charges the fastest and the $12 \mu \mathrm{m}$ configuration charges the slowest while the $16 \mu \mathrm{m}$ one is in between. But the $25 \mu \mathrm{m}$ configuration also saturates quickly and reaches the $2 \mathrm{~V}$ mark slower than the $16 \mu \mathrm{m}$ energy harvester. From the simulation results it can be foreseen that the $16 \mu \mathrm{m}$ configuration will also saturate at some point while the $12 \mu \mathrm{m}$ one reaches the highest voltage.

Similar results have been obtained from the voltage booster. Fig. 7 shows the charging waveforms of Type II microgenerator with $25 \mu \mathrm{m}$ coil connecting with 3,4 and 5 stages Dickson VMs. It can be seen that the energy harvester with 3 -stage VM charges the super capacitor to $2 \mathrm{~V}$ first and the one with 5-stage VM can reach highest voltage.

From the simulation results we can conclude that in an energy harvester design that combines electromagnetic microgenerator and voltage multiplier, the fewer number of turns in the coil and the fewer VM stages, the higher initial charging rate the energy harvester can get but the lower voltage it can finally reach. Therefore, although the micro-generator with more coil turns can generate more power and VMs with more stages can boost the voltage higher, under certain circumstances the optimisation of these subsystems in isolation does not lead to a globally optimised design. In the traditional 


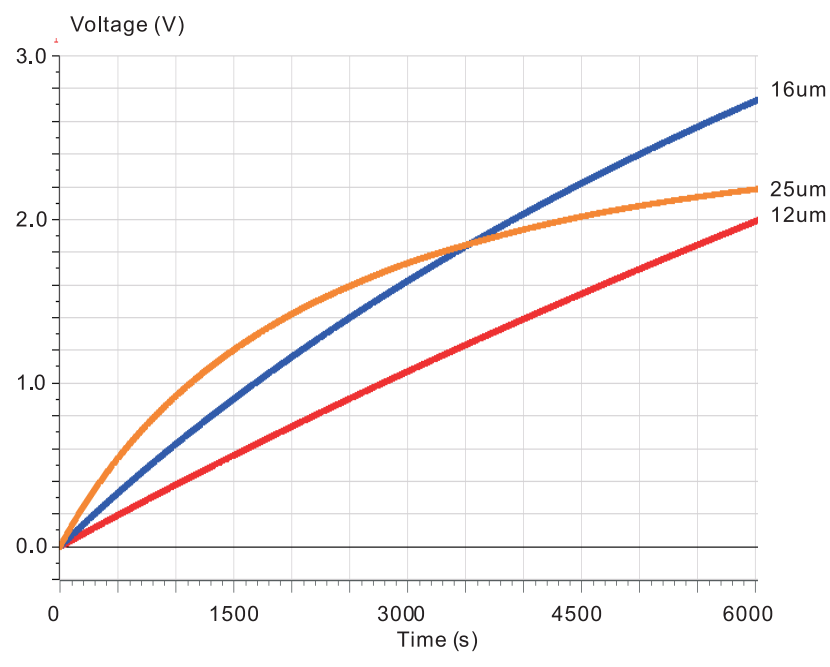

Fig. 6. Simulation of Type I micro-generator with different coils

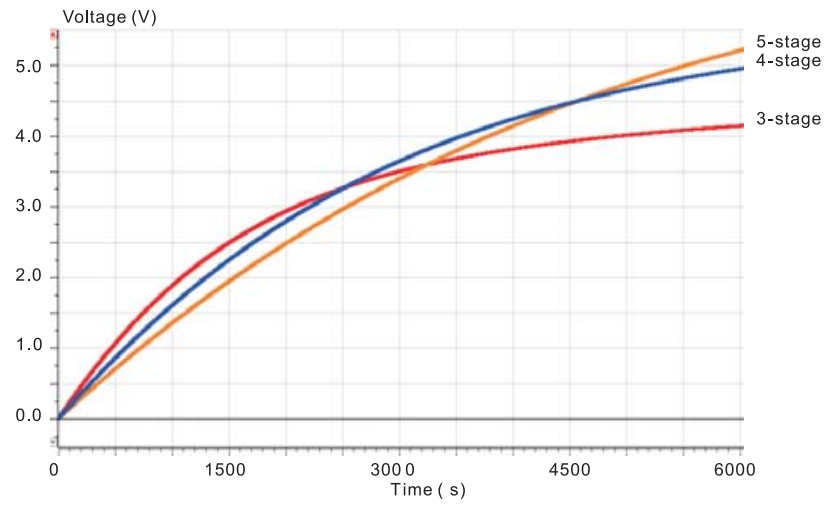

Fig. 7. Simulation of energy harvester with different VMs

approaches, where different components of an energy harvester in different physical domains are designed in separation, the gain at one part may come at the price of efficiency loss elsewhere, rending the energy harvester much less efficient than expected. Our results provide evidence that a holistic approach, which is based on an integrated design of the complete system with the micro-generator loaded by the associated electronics, can lead to superior performance characteristics and hence may be very useful for the development of future, more complicated systems and model libraries.

\section{PERFORMANCE OPTIMISATION}

In the proposed design flow (Fig. 2), the generated energy harvester configuration should be parameterised such that automated performance optimisation, carried out in the inner loop of Algorithm 1, will be able to further improve the energy harvester efficiency by employing suitable optimisation algorithms. The parameters used for the optimisation are from both the micro generator and the voltage booster. The optimisation object is to increase the voltage across the super capacitor. The optimisation algorithm generates design parameters to the model and obtains the charging rate through simulation. The optimisation loop runs continuously until the design parameters reach an optimum (Fig. 2). The micro-generator parameters that can be optimised are related to the coil size, i.e the thickness $(t)$ and the outer radius $(R)$. Because other components such as the magnets and cantilever determine the resonant frequency of the micro-generator and thus should be based on application requirements. The circuit parameters of voltage booster are the capacitor values of each VM stage. The entire energy harvester is optimised as an integrated model. The searching space of parameters is given in Table III. The optimisation is based on the concurrent simulations of design instances from uniform sampling the search space and track the best result. This is relatively simple and straightforward because after the automatic structure configuration the search space is quite small. However other optimisation algorithms may also be applied. We have demonstrated an integrated optimisation directly implemented in an HDL testbench which employs a genetic algorithm where at each genetic generation multiple instances of the design are simulated concurrently as individual chromosomes and analysed for selection [10]. To validate the effectiveness of our proposed approach to improve energy harvesting efficiency, we have carried out the following simulations and experimental measurements. The vibrationbased micro-generator is based on the cantilever structure and has been designed separately from the remaining parts of the energy harvester. The voltage booster circuit uses the optimal configuration published in literature [18] and has also been designed independently from the rest of the energy harvester. Parameters of original design are given in Table IV.

TABLE III

OPTIMISATION SEARCHING SPACE

\begin{tabular}{cc}
\hline Wire diameter(um) & $12 / 16 / 25$ \\
\hline Coil thickness $(\mathrm{mm})$ & $1.0-1.3$ \\
\hline Coil radius $(\mathrm{mm})$ & $2.0-2.45$ \\
\hline Capacitor values $(\mathrm{uF})$ & $47 / 100 / 150$ \\
\hline
\end{tabular}

TABLE IV

PARAMETERS OF ORIGINAL ENERGY HARVESTER

\begin{tabular}{c|c}
\hline \multicolumn{2}{c}{ Micro-generator } \\
\hline Wire diameter(um) & 16 \\
\hline Coil thickness(mm) & 1.3 \\
\hline Coil radius(mm) & 2.45 \\
\hline \multicolumn{2}{c}{ Voltage transformer } \\
\hline VM configuration & 5 -stage Dickson \\
\hline Capacitor values(C1-C5,uF) & $47,150,150,47,150$ \\
\hline
\end{tabular}

After the proposed design flow, Table $\mathrm{V}$ gives the new micro-generator and voltage booster parameters which are referred to as "optimised" design. The impact of these values on increasing the voltage across the super capacitor has been investigated in both simulation and experimental measurements (see Fig. 8). The impact of using the super capacitor model in Fig. 5 instead of an ideal capacitor has also been investigated. As can be seen from the figure, there is good correlation between the simulation and experimental waveforms in both the energy harvester designs, which validates the effectiveness and accuracy of the proposed design flow. Fig. 8 shows that 
the energy harvester from original design can charge the super capacitor to $2 \mathrm{~V}$ in 6000 seconds while the optimised design only uses 1500 seconds, which represents a $75 \%$ improvement.

TABLE V

PARAMETERS OF ENERGY HARVESTER CONFIGURED USING ALGORITHM 1

\begin{tabular}{c|c}
\hline \multicolumn{2}{c}{ Micro-generator } \\
\hline Wire diameter(um) & 25 \\
\hline Coil thickness $(\mathrm{mm})$ & 1.3 \\
\hline Coil radius(mm) & 2.0 \\
\hline \multicolumn{2}{c}{ Voltage transformer } \\
\hline VM configuration & 3-stage Dickson \\
\hline Capacitor values(C1-C3,uF) & $100,100,47$ \\
\hline
\end{tabular}

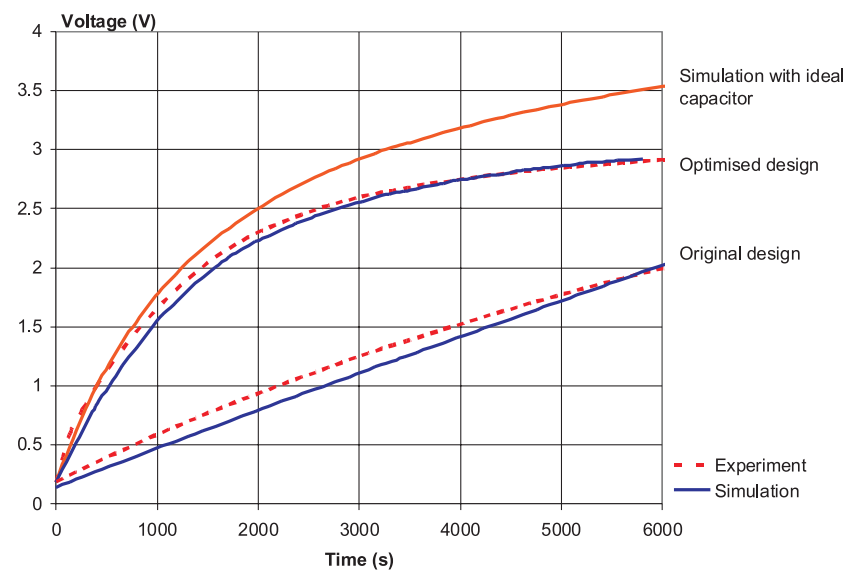

Fig. 8. Simulation and experimental waveforms of original and optimised energy harvesters

\section{CONCLUSION}

This paper presented an automated energy harvester design flow that can generate optimised configuration from an existing HDL model library as well as carry out performance optimisation through the employment of a single software platform. The effectiveness of the proposed design flow has been demonstrated by automatic structure configuration, optimisation and experimental validation of an energy harvester powered by an electromagnetic vibration-based micro-generator. We have shown that this approach can be used to investigate the tradeoffs between different energy harvester components where the optimisation of subsystems in isolation does not lead to a globally optimal design. A new energy harvester has been manufactured according to the outcome from the proposed design flow and experimental measurements of the new device have validated the results which show a 75\% improvement in the super capacitor charge rate. Continuing work will expand the design flow by investigating other types of micro-generator, such as piezoelectric and electrostatic, as well as new power processing circuit architectures. Further work will also focus on developing new optimisation algorithms that can speed up CPU time.

\section{ACKNOWLEDGMENT}

The authors would like to thank the Engineering and Physical Sciences Research Council (EPSRC-UK) for funding this work in part under grant number EP/E035965/1.

\section{REFERENCES}

[1] M. Schmitz, B. Al-Hashimi, and P. Eles, System Level Design Techniques of Energy-Efficient Embedded Systems. Kluwer Acad. Publishers, 2003.

[2] J. A. Paradiso and T. Starner, "Energy scavenging for mobile and wireless electronics," Pervasive Computing, IEEE, vol. 4, no. 1, pp. 1827, 2005.

[3] S. P. Beeby, M. J. Tudor, and N. M. White, "Energy harvesting vibration sources for microsystems applications," Measurement science and technology, vol. 17, no. 12, pp. R175-R195, 2006.

[4] T. von Buren, P. Mitcheson, T. Green, E. Yeatman, A. Holmes, and G. Troster, "Optimization of inertial micropower generators for human walking motion," Sensors Journal, IEEE, vol. 6, no. 1, pp. 28-38, 2006.

[5] P. Mitcheson, T. Green, E. Yeatman, and A. Holmes, "Architectures for vibration-driven micropower generators," Journal of Microelectromechanical Systems, vol. 13, no. 3, pp. 429-440, 2004.

[6] P. Mitcheson, P. Miao, B. Stark, E. Yeatman, A. Holmes, and T. Green, "MEMS electrostatic micropower generator for low frequency operation," Sensors and Actuators A: Physical, vol. 115, no. 2-3, pp. 523-529, 2004.

[7] M. El-Hami, P. Glynne-Jones, N. White, M. Hill, S. Beeby, E. James, A. Brown, and J. Ross, "Design and fabrication of a new vibration-based electromechanical power generator," Sensors and Actuators A: Physical, vol. 92, no. 1-3, pp. 335-342, 2004.

[8] S. Beeby, R. Torah, M. Tudor, P. Glynne-Jones, T. O’Donnell, C. Saha, and S. Roy, "Micro electromagnetic generator for vibration energy harvesting," J. Micromechanics and Microengineering, vol. 17, no. 7, pp. 1257-1265, 2007.

[9] R. Amirtharajah, J. Wenck, J. Collier, J. Siebert, and B. Zhou, "Circuits for energy harvesting sensor signal processing," in Design Automation Conference, 2006 43rd ACM/IEEE, 24-28 July 2006, pp. 639-644.

[10] L. Wang, T. Kazmierski, B. Al-Hashimi, S. Beeby, and R. Torah, "Integrated approach to energy harvester mixed technology modelling and performance optimisation," in Design, Test and Automation in Europe, 10-14 Mar. 2008, pp. 704-709.

[11] K. Gandu, P. Mitcheson, and T. Green, "Simulation toolkit for energy scavenging inertial micro power generators," in Proceedings of PowerMEMS 2007, 2007, pp. 137-140.

[12] E. Christen and K. Bakalar, "VHDL-AMS-a hardware description language for analog and mixed-signal applications," Circuits and Systems II: Analog and Digital Signal Processing, IEEE Transactions on, vol. 46, no. 10, pp. 1263-1272, Oct. 1999.

[13] Perpetuum Ltd, http://www.perpetuum.co.uk/.

[14] R. Torah, S. P. Beeby, M. J. Tudor, T. O’Donnell, and S. Roy, "Development of a cantilever beam generator employing vibration energy harvesting," in Proceedings of The 6th Int. Workshop on Micro and Nanotechnology for Power Generation and Energy Conversion Applications, 2006, pp. 181-184.

[15] H. Yan, J. Macias Montero, A. Akhnoukh, L. de Vreede, and J. Burghartz, "An integration scheme for rf power harvesting," in Proc. STW Annual Workshop on Semiconductor Advances for Future Electronics and Sensors, 17-18 November 2005, pp. 64-66.

[16] R. Nelms, D. Cahela, R. Newsom, and B. Tatarchuk, "A comparison of two equivalent circuits for double-layer capacitors," in Applied Power Electronics Conference and Exposition, 1999. APEC '99. Fourteenth Annual, 14-18 March 1999, pp. 692-698 vol.2.

[17] Mentor Graphics Corporation, SystemVision User's Manual, ser. Version 3.2, Release 2004.3, July 2004.

[18] R. Torah, M. Tudor, K. Patel, I. Garcia, and S. Beeby, "Autonomous low power microsystem powered by vibration energy harvesting," pp. 264-267, 28-31 Oct. 2007. 